\title{
Effect of Creep on Stability of Structural Elements
}

\author{
Alexandre de Macêdo Wahrhaftig* and Kaique Moreira Matos Magalhães \\ Polytechnic School, Federal University of Bahia (UFBa), Brazil
}

*Corresponding author: Alexandre de Macêdo Wahrhaftig, Department of Construction and Structures, Federal University of Bahia, Brazil.

Received Date: July 22, 2019

Published Date: July 26, 2019

\begin{abstract}
Creep, which represents the gradual increase of deformation over time, is a typical phenomenon of viscoelastic materials. Its consideration is necessary in the stability verification of compressed slender pieces in ultimate limit state because these pieces can have their stiffness modified as a function of the rheology of the material. Mathematically, creep can be characterized by models where the immediate elastic deformation is increased by viscous deformation, resulting in a temporal function. Although the first postulations for the understanding of stability (or buckling) have been solved statically, the phenomenon penetrates the field of structural dynamics since it involves the concept of the vibration of mechanical systems. In both cases, whether static or dynamic, it is necessary to consider the total stiffness of the structural element as composed of two terms, the conventional stiffness portion and the geometric stiffness portion. In this way, it is possible to include in the first plot a modulus of elasticity variable in time, that allows to follow the increase of the deformations, according to the model adopted for their representation, even under constant tension.
\end{abstract}

Keywords: Creep; Stability; Critical buckling load; Slender elements; Viscoelastic materials

\section{Introduction}

Slow deformation, or creep, is a type of time-dependent phenomenon, which is also related to loads and deformations, defined as the increase of deformation over time under the action of permanent loads or stresses. Creep produces permanent deformation, potentially altering the characteristics of the materials and their mechanical properties and can even lead to the collapse of a structure. A part of the strain produced by creep is recoverable, while another is residual and irrecoverable. Once a structural element is unloaded, an immediate recovery of the deformation occurs, which is approximately equal to that of the elastic deformation initially suffered. Another part of the deformation is slowly recovered. However, a third part of this deformation remains unrecoverable. Creep is associated with the rheological behavior due to the viscoelastic nature of certain materials, resulting in a continuous increase of the deformations even under constant tension. Although wood was had been the first material in which the effect of slow deformation was realized, it only began to be investigated when seen in other materials, such as concrete. Mathematically, creep can be represented by a timedependent function associated with viscoelastic rheological models capable of describing the phenomenon.

In this sense, the rheological models used to represent the viscoelastic behavior of many solids are usually based on the association of springs and dashpots that predict the total deformations and try to describe more appropriately the behavior of each material or group of materials. This behavior depends on, among other factors, the duration and time of application of loadings, and the creep can be classified into two types: basic creep and drying creep, the difference being in the existence or not of water exchange with the exterior. At the first one, it does not occur.

\section{Discussion}

From a practical point of view, technical standard codes take into account the phenomenon of creep in the dimensioning of structures by two considerations, either by proposing a coefficient of increase or reduction of stiffness or by proposing a coefficient of increase or decrease in resistance according to the time of application of the loading and moisture class. At the end, what is important to obtain is the function that will describe the deformation to be presented by the element over time, which arises from the calculation process. In the field of analysis, structural elements of the type beam/ column are characterized by being continuous systems which can sometimes be subjected to the action of axial compressive forces. When slender, these elements can reach an ultimate limit state defined by the loss of their stability, without having exhausted the resistant capacity of their sections. This condition is called buckling and is defined as the phenomenon in which a structural element 
loses its equilibrium or is bent under the action of a compressive axial force large enough to remove it from its initial straight configuration, this being the best known criterion for the safety verification of slender pieces, whose solution originated in the field of statics in rational mechanics. However, buckling is essentially a problem of a dynamic nature, since it involves the equilibrium of mechanical systems, dependent on stiffness and masses. Whether statically or dynamically, the stability verification of the slender elements should take into account all the changes undergone by the material over the life of the system, including creep.

This inclusion, either by the static or dynamic path, can be made by observing the stiffness of the structure, which must be composed of two terms, one corresponding to the portion of conventional stiffness and another to the geometric stiffness portion. In this way, it is possible to adapt the first one, introducing a variable modulus of elasticity over time, that allows to accompany the increase of the deformations according to the adopted criterion: normative or rheological model. Thus, the total stiffness takes the form in which the mathematical model used to represent the creep is introduced in the first part, via modulus of elasticity, and the second one is the geometric, that is a function of the normal force acting on the system. For further information on the concepts presented, it is suggested to observe the works [1-8].

\section{Conclusion}

The stability of slender elements is typically a phenomenon of a dynamic nature, although the first postulations have been originated elaborated in the static field. In any field, for the correct analysis of these systems, all aspects involving the stiffness of the structural elements, including the viscoelastic behavior of the material, must be considered. In so doing, the problem of stability is solved at the instant of interest, that is, it becomes a function of time. Based on this procedure, studies already performed indicate important reductions, of the order of $21 \%$, in the vertical loading capacity of a structure after stabilizing the deformations by creep.

\section{Acknowledgement}

None.

\section{Conflict of Interest}

No conflict of interest.

\section{References}

1. Wahrhaftig AM, Brasil RMLRF, Balthazar JM (2013) The first frequency of cantilever bars with geometric effect: A mathematical and experimental evaluation. J Braz Soc Mech Sci Eng 35(4): 457-467.

2. Wahrhaftig AM, Brasil RMLRF, Cesar SF (2016) Creep in the fundamental frequency and stability of a slender wooden column of composite section. Rev Árvore 40(6): 1119-1130.

3. Wahrhaftig AM, Brasil RMLRF (2016) Representative experimental and computational analysis of the initial resonant frequency of largely deformed cantilevered beams. Int J Solids Struct 102-103: 44-55.

4. Wahrhaftig AM, Brasil RMLRF (2016) Vibration analysis of mobile phone mast system by Rayleigh method. Appl Math Modell 42: 330-345.

5. Wahrhaftig AM, Brasil RMLRF (2017) Initial undamped resonant frequency of slender structures considering nonlinear geometric effects: the case of a 60.8 m-high mobile phone mast. J Braz Soc Mech Sci Eng 39(3): 725-735.

6. Wahrhaftig AM, Brasil RMLRF, Nascimento LSMSC (2018) Analytical and mathematical analysis of the vibration of structural systems considering geometric stiffness and viscoelasticity. In: Numerical Simulations in Engineering and Science.

7. Wahrhaftig AM (2019) Analysis of the first modal shape using two case studies. Int J Comput Meth 16(06): 1840019.

8. Wahrhaftig AM, Silva MA, Brasil RMLRF (2019) Analytical determination of the vibration frequencies and buckling loads of slender reinforced concrete towers. Lat Am J Solids Struct 16(5): e196. 